\title{
Sample similarity analysis of angles of repose based on experimental results for DEM calibration
}

\author{
Yuan Tan ${ }^{1, *}$, Willibald A. Günthner ${ }^{1}$, Stephan Kessler $^{1}$ and Lu Zhang ${ }^{2}$ \\ ${ }^{1}$ Institute for Materials Handling, Material Flow and Logistics \\ Technical University of Munich, Boltzmannstraße 15, 85748 Garching, Germany \\ ${ }^{2}$ Institute for Sensing Control Measurement and Precision Machining Technology \\ Dalian University of Technology, Linggong Road 2, Ganjingzi District, Dalian, Liaoning
}

\begin{abstract}
As a fundamental material property, particle-particle friction coefficient is usually calculated based on angle of repose which can be obtained experimentally. In the present study, the bottomless cylinder test was carried out to investigate this friction coefficient of a kind of biomass material, i.e. willow chips. Because of its irregular shape and varying particle size distribution, calculation of the angle becomes less applicable and decisive. In the previous studies only one section of those uneven slopes is chosen in most cases, although standard methods in definition of a representable section are barely found. Hence, we presented an efficient and reliable method from the new technology, 3D scan, which was used to digitize the surface of heaps and generate its point cloud. Then, two tangential lines of any selected section were calculated through the linear least-squares regression (LLSR), such that the left and right angle of repose of a pile could be derived. As the next step, a certain sum of sections were stochastic selected, and calculations were repeated correspondingly in order to achieve sample of angles, which was plotted in Cartesian coordinates as spots diagram. Subsequently, different samples were acquired through various selections of sections. By applying similarities and difference analysis of these samples, the reliability of this proposed method was verified. Phased results provides a realistic criterion to reduce the deviation between experiment and simulation as a result of random selection of a single angle, which will be compared with the simulation results in the future.
\end{abstract}

\section{Introduction}

According to the criterion of physical properties, granular material can be considered in a state between fluid and solid [1] and is able to maintain a static and stable condition with steepest angle of a classical conical sand-pile. The so-called angle of repose (AoR) is used to describe the flow behaviour and properties of granular material. At which, the material on the slope face is on the verge of sliding. As one of the most primary macroscopic parameters, AoR is widely studied and employed to calculate other parameters of granular material or indirectly describe solids motion, for example, calibration of material parameter of discrete element method (DEM) simulation.

To measure AoR, many methods are available and at least four basics of them are frequently applied: loading simulated measuring box, bottomless cylinder, rotary drum with frictional walls and free falling in a measuring device. All of which were summarized in [1]. In the first method, AoR is measured under in-situ static pressure, while it is measured after a dynamic flow process in the other three methods.

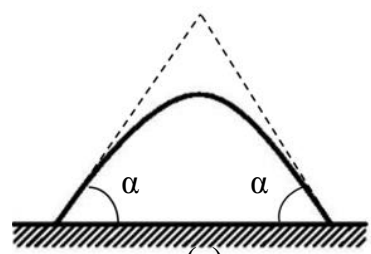

(a)

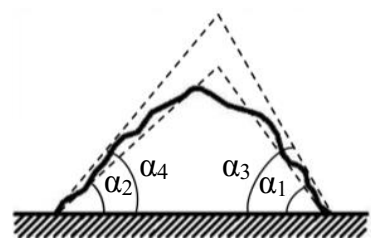

(b)

Fig. 1. Deviations of the formed willow chips heap from geometric cone solid: (a) cone with arc-shaped slope and linear generating lines; (b) cone with uneven slope and convex/concave generating line

Even though the measurement of AoR is predefined according to FEM [2] over several decades, just like the angle $\alpha$ pictured in Fig. 1 (a). In reality, the granular material forms an ideal cone shape like Fig. 1 (a) only in few cases. The results could widely range because of

\footnotetext{
* Corresponding author: $\underline{\tan @, \text { fml.mw.fml.de }}$
} 
particle characteristics (different shapes, wide size distribution) and material properties (e.g. sliding/rolling friction coefficient). In a previous study by Fraczek [3], three types of truncations (truncation of the top, cone with convex generating line and cone with concave generating line) were discussed for undesirable cones. Even though to measure extremely asymmetrical slope, such as Fig. 1 (b), either convex or concave generating line could also result in various AoR. The Angle $\alpha_{2}$ was greater than $\alpha_{1}$ in the case with concave generating line and the third case, and $\alpha_{3}$ smaller than $\alpha_{4}$ in the second case. Since it is generally reported that AoR increases with increasing sliding and rolling friction coefficients and deviation from spheres $[4,5]$, various angles would eventually lead to different calibrated material model for DEM simulation.

In light of the introduction above, predictable deviation between experiment and simulation by using traditional method could be even large in some extreme situations. As prescribed in [3], the difference of AoR between experiment and simulation should be within $\pm 5 \%$. We expect an initiate assessment process to minimize this deviation.

\section{Material and method}

Willow chips were chosen as the object of interest. Among all common bulk materials, it can build smooth top of heap and even arc-shaped surface because of the inhomogeneous particle size and low rolling as well as low sliding friction. As mentioned before, this kind of heap results in lower accuracy of calibration of material model. The experimental set-up, bottomless cylinder, consists of two essential parts (a cylindrical container and lifting equipment) and an extra rotatable plate.

\subsection{Experiments}

In the present study, measurements of AoR were conducted using a motor-driven bottomless cylinder with two dimensions, the inner diameter was $425 \mathrm{~mm}$ and $250 \mathrm{~mm}$ and the height was $595 \mathrm{~mm}$ and $350 \mathrm{~mm}$, respectively. Biomass materials were lifted in two different velocities $(142 \mathrm{~mm} / \mathrm{s}$ and $33 \mathrm{~mm} / \mathrm{s})$ with three repetitions, respectively. For the purpose of $3 \mathrm{D}$ reconstruction of the formed heap, the new Xbox Kinect V2 sensor was utilized. Although the Kinect V2 was not designed for rehabilitation specifically. However, it allows free interaction between controller and full 3Drecognition $[6,7]$. In total 12 independent experiments were completed. Since the form of heaps didn't reveal significant difference among them, the following content will be continued with only one experiment as example.

Two different views of the heap during one experiment are shown above (Fig. 2). As has been described earlier, the measurement of AoR is characterized by a considerable inaccuracy as a result of deviations of the shape of the cone generatrix from a straight line. Therefore, divergences of AoR of this same sand-pile suggested the poor quality of traditional methods.

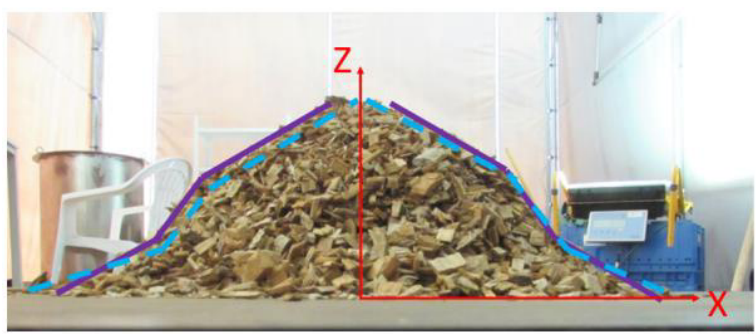

(a)

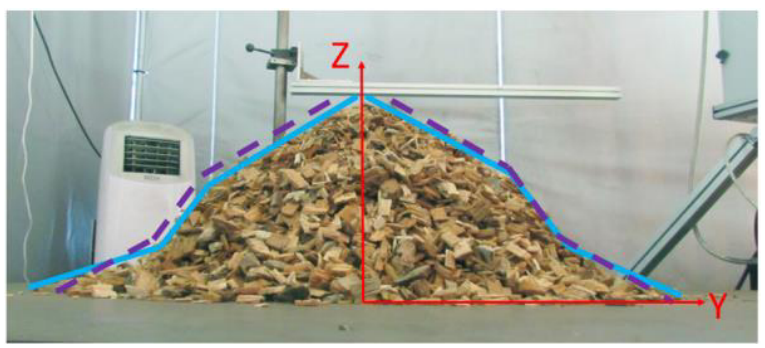

(b)

Fig. 2. Photos of the heap: (a) purple solid line-contour of view $\mathrm{XZ}$; (b) blue solid line-contour of view YZ.

\subsection{Data processing}

Prior to data processing, the generated data need to be pre-processed in order to achieve desired accuracy within acceptable computation time. Specifically, overlapped and excessive points were removed by using MeshLab $^{\circledR}$ [8] so that the file size was 100 times minimized.

The data processing of the surface of repose consists of six basic processes:

1. The scanned surface is obtained in STL format, thereby generating point clouds in Matlab R2015b ${ }^{\circledR}$ [9] (see Fig. 3 (a)).

2. The approach proceeds with analysis of each single slice, which consists of two parallel, $5 \mathrm{~mm}$ apart, planes (see Fig. 3 (a)). Since $5 \mathrm{~mm}$ is negligible compared to the dimension of the heap, it was hypothesized that all points are located approximately at the same tangential plane of the conical surface. The position of slice may not be precisely determined but two planes must symmetric to a same vertical plane $\left(\mathrm{P}_{\mathrm{m}}\right)$, which passes through the origin. Thus, the points in each slice are successfully isolated (see Fig. 3 (b)).

3. These selected points are projected onto plane $\mathrm{P}_{\mathrm{m}}$, as shown in Fig. 3 (c).

4. An inclined line, which forms AoR with the horizontal line, need to be generated based on these scatter-plots. Then a LLSR technique was applied to the linearized regression (see Fig. 3 (d)).

5. Two angles of repose are computed for each slice.

6. Two planes of the first slice are rotated simultaneously around the origin of coordinate system to obtain new slices of the heap (see Fig. 3 (e)). Then, steps 3-5 are repeated.

These steps are schematically represented below. Following the steps, a great number of samples of AoR were studied. Here in this work, 17 slices, namely 34 angles were calculated. Based on statistic 


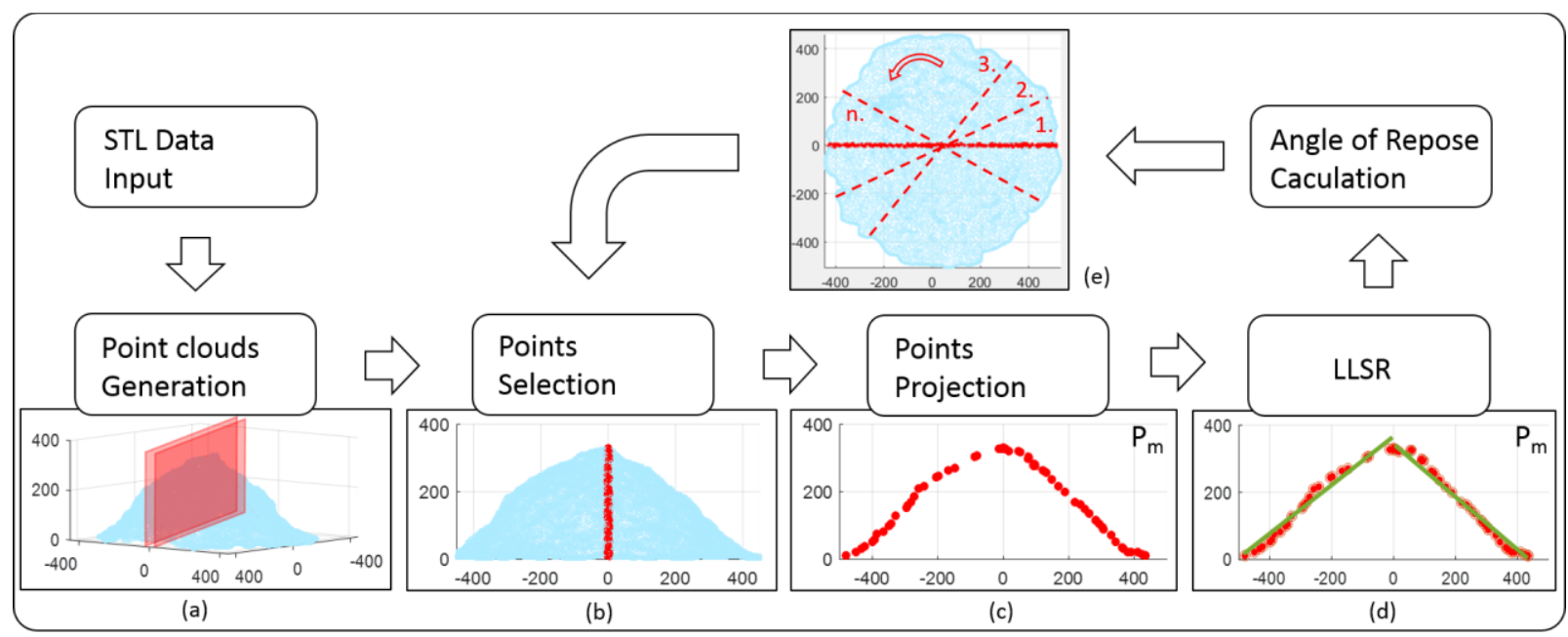

Fig. 3. Date processing of point cloud.

theory, the more samples and angles in each sample are, the more accurate analysis is in practice. Otherwise, the proposed algorithm was started with a low-density point clouds, in other words, coarse STL file. For sure it will be also feasible with intensive point clouds for the sake of more accurate analysis in actual applications, yet at the expense of large memory and computational effort.

\section{Results and discussion}

\subsection{Correlation coefficient of linear regression}

According to the trend of the selected points, the surface was considered as a linear regression in the whole calibration range, as shown in Fig. 4. To determine whether the regression equation truly represent the set of data, the correlation coefficient $(r)$ was examined using the following mathematical formula [10]:

$$
r=\frac{n \sum x y-\left(\sum x\right)\left(\sum y\right)}{\sqrt{n\left(\sum x^{2}\right)-\left(\sum x\right)^{2}} \sqrt{n\left(\sum y^{2}\right)-\left(\sum y\right)^{2}}}
$$

Herein, the linear regression of only one sample is presented. Fig. 5 indicates that $x$ and $y$ of all samples have a strong linear correlation, with the absolute values of $r$ extremely close to 1 . The results of other samples were similar. In addition, the fitting lines could be more accurate using obtained overlapping plots from uncompressed STL file.

The two dashed lines in two diagrams of Fig. 4 represent different supposable measurements. Therefore, compared to angle $\left(\beta_{1}\right.$ and $\left.\beta_{2}\right)$ of the linear regression line, these two lines are clearly deviated from each other in two cases: (1) the line was drawn tangentially to the points of lower part of the slope (angle $\beta_{11}$ and $\beta_{21}$ ); (2) the line was drawn tangentially to the upper part (angle $\beta_{12}$ and $\beta_{22}$ ). The difference between each two angles of dashed lines is over 5\%, approaching the highest allowable limit for calibration. As a result, it could lead to distinct material models of DEM after calibration, if any of them is regarded as reference.

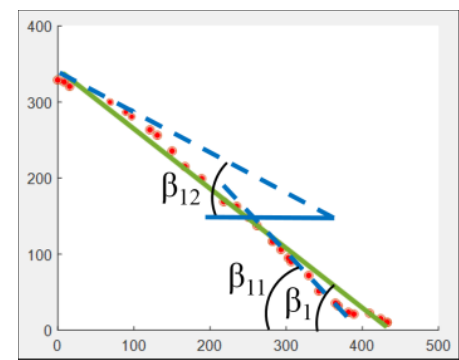

(a)

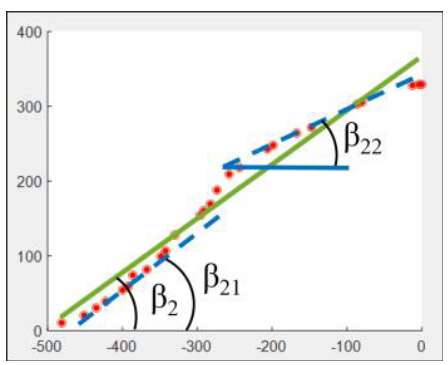

(b)

Fig. 4. Fitting lines according to LLSR and convex/concave generating lines of the selected section (a: right slope; b: left slope)

\subsection{Sample similarity analysis}

The proposed computational approach provides a way of obtaining a sample of AoR from the whole surface of heap, instead of one single AoR. In the next step, as mentioned in our objective, the similarity of different samples was investigated. Therefore, both the mean value and the standard deviation of each sample, were calculated to quantify the amount of variation or dispersion of a set of data values.

The mean values and standard deviations of 10 samples, each including 34 approximatively computed angles, are presented in Fig. 6. The values of them range from 37.4 to 37.75 and from 1.89 to 2.46 respectively.

Considering both of them could be taken as criterion to assess the similarity of heap, with closed mean values and low standard deviation it could be concluded, that the results show the efficiency of the proposed method. 


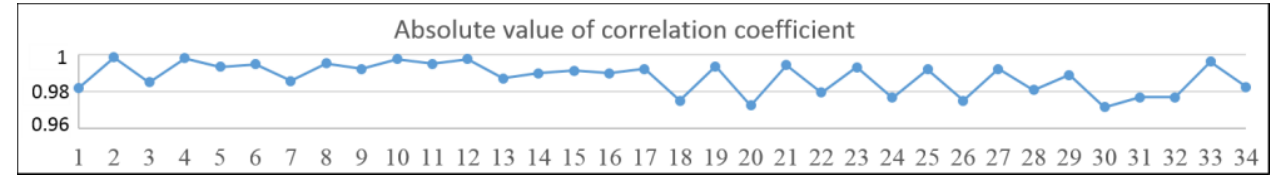

Fig. 5. Absolut value of correlation coefficient of LLSR (one sample).

As mentioned in section 2.2 , every slice was located randomly and independently from each other. The result of similarity analysis demonstrated that any sample of AoRs of the same heap is able to characterize the slope, which is directly related to material parameters.

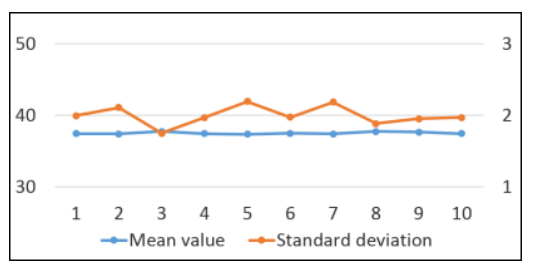

Fig. 6. Mean value and standard deviation of AoRs.

\section{Conclusion}

We believe that the proposed method, based on experimental evaluation, has a number of potential applications in the representation and expression of sand-pile of granular material with entire threedimensional data. This study demonstrate that the Xbox Kinect V2 is a useful and cost-effective devise, which featured simple operation, time saving, and high performance.

Furthermore, this data processing procedure may be used to analyze the sand-pile form of simulation as well. In this way the DEM calibration could be accomplished by analyzing two random selected set of angles of experiment and simulation, instead of comparing two single angle of both processes.

\subsection{Major advantages}

Based on the result of this study, the proposed method is applicable to general problems and especially for cases with arc-shaped top or uneven slope.

As stated at the beginning of section 2.1, 12 tests were carried out. The similarity analysis of samples of AoRs for each test suggests a high repeatability regardless of objective factors, such as cylinder size, and lifting velocity, or subjective factors, like choice of view and point to measure as well as visual estimation about the tangent line of slope. As the physical performance of material is assessed based on the entire form of heap, namely, a sample of enough angles, instead of twodimensional aspect.

It can be predicted that the propose method could also be applied in prospective analysis of simulation with bottomless cylinder. Hence, calibration of material parameter will be accomplished based on comparison between two samples of angles random selected from experiment and simulation. Furthermore, errors induced by objective and subjective factors could be considerably reduced.

\subsection{Comparison with conventional methods}

Unlike conventional methods, this method requires 3Drecognition instrument, which can obtain assessable data format of the surface of sand-pile at least. However, for some types of materials, the shape is somewhat angular and the particles have large size distribution range, which may build bridge during the lifting process, so that the correlation coefficient may not meet the requirements of linear regression. Therefore, further work is expected to enhance the applicability of the method for calibration of material parameters.

\section{Outlook}

In comparison with other traditional methods the proposed method, especially data processing and afterwards, is much more complex than conventional methods. The corresponding graphical user interface (GUI) is on-going, which enhances the efficiency and ease of use for the underlying logical design of stored program. Instead of text-based programming, such as typed command labels and text navigation, the automatic measurement analysis only requires data input from Kinect and simple icons. To facilitate calibration of material parameter, the ultimate goal is to find out a practicable assessment method. So the analysis of the similarity as well as difference between experiment and simulation should be performed in future studies.

\section{References}

[1] A. Wu, Y. Sun, Granular dynamic theory and its applications. 17 (2008).

[2] The European Federation of Materials Handling "FEM", FEM 2.582, General properties of bulk materials and their symbolization, (1991)

[3] J. Frączek, A. Złobecki, J. Zemanek, Journal of food engineering, 83, 17. (2007)

[4] Y. C. Zhou, B. H. Xu, A. B. Yu, Zulli, P. Powder technology, 125, 45 (2002)

[5] W. Wang, J. Zhang, S. Yang, H. Zhang, H. Yang, G. Yue, Particuology, 8, 482 (2010)

[6] ST. Smith, D. Schoene, Aging Health 2012, 8, $243-$ $52(2012)$

[7] MJD. Talyor, D. McCormick, T. Shawis, R. Impson, Res Dev 2011, 48, 1171-1186 (2011).

[8] Meshlab, version V1.3.3 ISTI (CNR research center, Pisa, Italien, 2015)

[9] Matlab, version R2014b (The MathWorks Inc, Natick, Massachusetts, 2014)

[10]Bland, J.M., Altman, D.G, Statistics notes: measurement error. BMJ. 312 (7047), 1654 (1996) 